\title{
Morphology and biomechanics of the nests of the Common Blackbird Turdus merula
}

\author{
LUCIA E. BIDDLE, D. CHARLES DEEMING* and ADRIAN M. GOODMAN \\ School of Life Sciences, University of Lincoln, Joseph Banks Laboratories, Lincoln, LN6 7DL, UK
}

Capsule Common blackbirds select different materials, with varying biomechanical properties, to construct different parts of their nest.

Aims This study tested the hypothesis that outer components of a nest have a more structural role and so are stronger than materials used to line the cup.

Methods Blackbird nests were measured prior to being dismantled to isolate structural components which were tested for mechanical strength and rigidity.

Results Outer nest wall materials were significantly thicker, stronger and more rigid than materials in the inner structural wall or the cup lining. In the vertical plane materials used in the structural wall did not differ. By contrast, lining materials from the bottom of the nest cup were significantly thicker, stronger and more rigid than materials from the top of the cup.

Conclusion Blackbirds use different materials in nest construction roles suited to their properties and so may be able to recognize the structural properties of these materials. Materials on the outside of the nest may have a key structural role during construction.

Bird nests have long been considered remarkable structures serving as sites for incubation of eggs and in many species, rearing of chicks. Whilst we know in general terms what nests are constructed of (Hansell 2000, Ferguson-Lees et al. 2011) there are relatively few reports that quantify the materials used (Rohwer \& Law 2010, Britt \& Deeming 2011, Álvarez et al. 2013, Mainwaring et al. 2014). Detailed nest construction behaviour is rarely described, but see Skutch (1976), Collias \& Collias (1984) and Hansell (2000), but where this is the case, the materials used seem to have been chosen with care and the method of construction can be quite precise (Hansell 2000, Bailey et al. 2014). Male Southern Masked Weaver Birds Ploceus velatus weave complex nests from blades of grass but sequential nests built by the same male vary significantly in structure (Walsh et al. 2010, 2011). By contrast, Penduline Tits Remiz pendulinus exhibit a high degree of repeatability in nest mass (Schleicher et al. 1996). Other studies have confirmed that nest construction exhibits a high degree of plasticity particularly in response to prevailing temperature during nest construction (Britt \& Deeming 2011, Deeming et al. 2012, Mainwaring et al. 2012, 2014).

${ }^{*}$ Correspondence author: Email: cdeeming@lincoln.ac.uk
Such observations imply that behaviour of a nesting bird is adaptive with respect to the physical requirements of its nest's structure and function. Choice of materials and their subsequent placement in different parts of the nest are presumably decisions made by the bird as it constructs the nest. It is possible to define various layers of a nest according to their presumed role (Hansell 2000). These vary from an outer layer that may function primarily in terms of crypsis through to an innermost layer, which may primarily function in terms of insulation (Hansell 2000). For instance, Long-tailed Tits Aegithalos caudatus cover their nests with lichens, which are seen as a form of camouflage (McGowan et al. 2004) whereas cavity nesting Blue Tits Cyanistes caeruleus line their nest cups with feathers and fur, which serve as effective insulators (Mainwaring \& Hartley 2008). Nests are often substantial structures that have to hold securely not only the mass of the clutch of eggs but also the incubating birds, and in small passerine species often broods of nearly fledged chicks that each may exceed the body mass of the adults (Deeming 2013).

As well as parts of the nest serving camouflage and insulation functions (Hansell 2000) it is reasonable to assume that there are parts of a nest that would have a more structural role, for instance, nest attachment to a 
substrate (Hansell 2000). For instance, elements used within the structural nest layer forming the bulk of a nest wall presumably will have a high degree of strength and stiffness to offer physical support. By contrast, lining materials within a cup should be more flexible. Recent work on nest construction in primates has shown the importance of the mechanical role of nest elements and its significance in terms of nest building behaviour. van Casteren et al. (2012) demonstrated that a compliant central structure, with thicker more rigid and stronger outer elements, is optimal for safely supporting the weight of sleeping Orangutans Pongo species. However, different parts of bird nests may have more than one role. Recently, construction of Australian Passerine nests has been linked more with a structural function than insulation (Heenan \& Seymour 2011) but without measurement of structural properties. Resolving whether nests are primarily built by birds to maximize crypsis, insulation or structural requirements, or a combination of these factors, will help us better understand the evolution and functional properties of nests. We may then be able to measure how climate change may impact on nest construction and the reproductive biology of various bird species (Mainwaring et al. 2012, 2014).

The study described here takes the first step to better understand the biomechanical characteristics of the components of a bird nest in order to better understand the roles that the component materials may play. To do this we study nests of the Common Blackbird Turdus merula, which are of interest because they are one of few species in which nests have been quantified in terms of their materials and insulatory properties: the thermal properties of these nests are only closely correlated with the amount of dried grass (Mainwaring et al. 2012). However, the plant materials in the outermost layers of the Blackbird nest may be important in crypsis or have key structural roles. We hypothesized that components of the outer structural wall have important structural roles rather than simply serving to camouflage the nest. Hence, we predicted that the elements contributing to the structural wall would be stronger and more rigid than those used in the lining.

\section{METHODS}

Twelve nests of the Common Blackbird T. merula were collected after nest abandonment at the end of the breeding season over several years from various locations in Lincolnshire, Nottinghamshire and Hertfordshire,
UK. The nests had been constructed by different females although the exact times of construction were unknown but presumed to be in the spring. Nests were stored for at least a year at approximately room temperature and humidity before analysis. Detailed observations of the arrangement of elements within the nest and identification of key structural regions were based upon observations and previous studies (Heenan \& Seymour 2012, Mainwaring et al. 2014). Nests were conditioned prior to testing at $23^{\circ} \mathrm{C}, 50 \% \mathrm{RH}$ for 2 weeks in an environmental chamber (Sanyo MLR351H, Osaka, Japan).

The dimensions of the complete nests were measured in two planes both parallel and perpendicular to the long axis of the nest; wall thickness and depth measured using callipers. The nest was weighed using a top pan balance (Sartorius CP3202s, Goettingen, Germany) and the volume of the nest cup was determined by two methods in order to compare techniques. Firstly, the cup was lined with commercial cling-film before filling the space with $4.76 \mathrm{~mm}$ diameter acrylic beads, which were emptied out before dental clay was added (Dentstone KD Plaster, BPB Formula, Newark, UK). The mass of each type of material was determined and predetermined density values were used to calculate volume. Thereafter, the nests were carefully dismantled to obtain samples for the mechanical tests.

Three main cup-shaped regions were identified from the outer edge inwards to the centre and termed: outer nest (loosely arranged, generally not interwoven), structural wall (sometimes interwoven, typically incorporating mud, cup-like) and the cup lining (interwoven structure, cup-like shape). The nests were elliptical in shape and so zones perpendicular and parallel to the longest axis of the nest were compared. Changes within the vertical profile of the nest were also investigated by testing elements from vertical locations from the rim to the base of the nest. These were defined as: the top wall measuring $0-2 \mathrm{~cm}$ from the top rim; mid nest wall measuring $2-4 \mathrm{~cm}$ from the rim and basal nest wall measuring more than $4 \mathrm{~cm}$ from the upper rim for the whole nest. The cup lining was divided into the top $0-2 \mathrm{~cm}$ from the rim and the base was below this.

A detailed mechanical analysis of the construction elements within the nest was undertaken in order to relate the composition of the nest regions to their function. Each structural region was carefully deconstructed taking particular care to avoid damaging any of the individual elements, which consisted mainly of plant root and stem material. 
Structural elements from each region were arranged in order of increasing diameter. The six thickest elements from each region both parallel and perpendicular to the long axis of the nests underwent three-point bending tests using an Instron universal testing machine fitted with a $100 \mathrm{~N}$ load cell (model 4443, Instron, UK). Tests were carried out on the first $50 \mathrm{~mm}$ of each sample and the diameter was measured at the midpoint using a digital micrometer (Mitutoyo, Japan). The sample was placed between two supports and to limit the effects of shear the supports were set a sufficient distance apart to provide a minimum span-to-depth ratio of 20 (Vincent 1992). A pushing probe of radius $5 \mathrm{~mm}$ was attached to the load cell and lowered until it just touched the sample. The crosshead was then lowered at a rate of $10 \mathrm{~mm} \mathrm{~min}^{-1}$, causing the sample to bend until it eventually failed. An interfaced computer produced a graph of force versus displacement, permitting calculation of the mechanical properties of the sample, e.g. the bending strength, $M$ (Equation 1), and bending rigidity, EI (Equation 2). Data from samples which slipped from the supports during testing were excluded from the analysis.

The mechanical properties of each of the samples were calculated using the following equations (Gordon 1978). Bending strength, or maximum bending moment, $\mathrm{M}(\mathrm{N} \mathrm{m})$, is given by the expression:

$$
M=\frac{F_{\max } L}{4},
$$

where $F_{\max }$ is the maximum force $(\mathrm{N})$ a sample will withstand before it fails and $L$ is the distance $(\mathrm{m})$ between the supports. The bending rigidity, EI $\left(\mathrm{N} \mathrm{m}^{2}\right)$, of a uniform beam is the resistance of that beam to curvature and is given by:

$$
E I=L^{3}\left(\frac{\mathrm{d} F}{\mathrm{~d} \delta}\right) / 48
$$

where $\mathrm{dF} / \mathrm{d} \delta$ is the initial slope of the force displacement curve $\left(\mathrm{N} \mathrm{m}^{-1}\right)$.

For structural properties, the analysis was carried out using a single datum, which was the mean of the samples of regions with a minimum of three samples. Unfortunately, robust data could not always be collected from all of the six samples, which meant that all variables could not be recorded from all nests. Therefore, sample sizes were different according to the part of the nest being investigated: 11 nests for the structural components of the whole nest, 8 for the various vertical parts of the structural wall and 9 for the cup lining.

The resulting means were tested for normality (Anderson-Darling test). Strength $(M)$ and rigidity (EI) data were $\log _{10}$-transformed to normalize the data, which was confirmed statistically, prior to further analysis. Given that the nests were elliptical it was felt that it was appropriate to compare materials from both perpendicular and parallel to the long axis of the nest for the different regions in the top and middle of the nest. Paired $t$-tests, however, revealed no significant differences $(P>0.05$ in all cases) and so the values per nest were averaged prior to analysis. One-way ANOvA was used on MINITAB (version 15) to compare the effect of horizontal or vertical position with nest as a random factor. In the case of a significant effect of the factor post hoc Tukey tests were used to compare the various classes.

\section{RESULTS}

\section{Nest appearance and dimensions}

Common Blackbird nests are structures with an outer wall, composed of interwoven twigs, roots and leaves, and surrounding a cup lined with dry grass. The mud that contributes much of the mass of the nest is only evident once the outer and inner layers are stripped away (Fig. 1). It forms a substantial cup in which plant material has been incorporated.

The nests had one axis approximately $9 \%$ longer than the other axis perpendicular to it (Table 1). The maximum height of the nest was around two-thirds of its diameter (Table 1). Inner wall thicknesses measured at four points around the rim of the cup were not significantly different from each other $\left(F_{3,44}=0.3\right.$, $P=0.85)$ and so an average value is shown in Table 1 . The outer wall thickness was almost twice that of the inner wall thickness (Table 1).

Cup diameters were also asymmetrical with the longer axis being around $16 \%$ longer than the axis perpendicular to it (Table 1). The long axis for the cup and the nest were in the same orientation. Ratios for maximum-to-minimum nest diameters and cup diameters were not significantly different (paired $t$-test: $t_{11}=1.5, P=0.16$ ). Cup depth was around two-thirds of the cup diameter so that the ratio of cup depth to radius ranged between 1.171 and 1.355 .

Mean total nest mass was $163.8 \mathrm{~g}$ (Table 1) with a coefficient of variation of $30 \%$. The three structural 


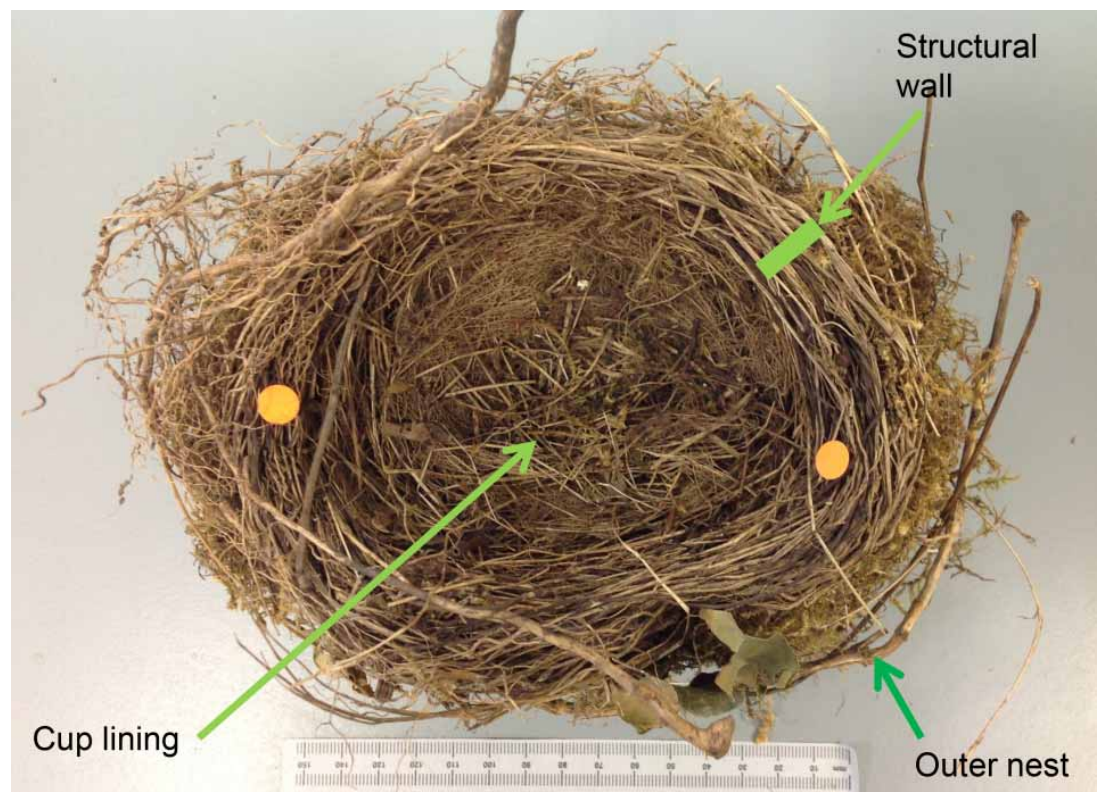

Figure 1. A blackbird nest from above with arrows indicating the position of the outer nest layer, the structural layer and the cup lining. Orange dots indicate ends of longest axis of the nest. Ruler indicates $\mathrm{mm} / \mathrm{cm}$ scale.

components varied in mass with the nest lining having the lowest mass $(<10 \%$ of the total) and the structural wall having the highest mass due to the high proportion of mud (Table 1). However, it was the mass of the outer wall that exhibited the greatest variation between nests (coefficient of variation $=93.2 \%$ ).

The volume of the nest cup determined using dental clay was $351.2 \mathrm{~cm}^{3}$, which was significantly larger, by $7.6 \%$, than the value determined using acrylic beads (Table 1; paired $t$-test: $t_{11}=-2.5, P=0.03$ ).

Table 1. Structural dimensions and properties of Common Blackbird Turdus merula nests. $n=12$ in all cases.

\begin{tabular}{lc}
\hline Variable & Mean $\pm \mathrm{sd}$ \\
\hline Nest diameter parallel to long axis $(\mathrm{mm})$ & $164.3 \pm 10.9$ \\
Nest diameter perpendicular to long axis $(\mathrm{mm})$ & $152.6 \pm 23.4$ \\
Ratio of nest diameters & $1.096 \pm 0.154$ \\
Nest height $(\mathrm{mm})$ & $104.5 \pm 11.7$ \\
Mean outer wall thickness $(\mathrm{mm})$ & $29.5 \pm 7.1$ \\
Mean structural wall thickness $(\mathrm{mm})$ & $17.4 \pm 4.3$ \\
Cup diameter parallel to long axis $(\mathrm{mm})$ & $106.8 \pm 7.5$ \\
Cup diameter perpendicular to long axis $(\mathrm{mm})$ & $92.3 \pm 9.6$ \\
Ratio of cup diameters & $1.165 \pm 0.107$ \\
Maximum cup depth (mm) & $61.8 \pm 9.2$ \\
Total nest mass $(\mathrm{g})$ & $163.8 \pm 50.4$ \\
Outer wall mass $(\mathrm{g})$ & $58.6 \pm 54.8$ \\
Structural wall mass $(\mathrm{g})$ & $90.8 \pm 37.6$ \\
Cup lining mass $(\mathrm{g})$ & $14.3 \pm 4.9$ \\
Cup volume - dental putty $\left(\mathrm{cm}^{3}\right)$ & $351.2 \pm 50.2$ \\
Cup volume - acrylic beads $\left(\mathrm{cm}^{3}\right)$ & $326.1 \pm 41.1$ \\
\end{tabular}

\section{Mechanical properties}

Analysis of the pooled data for each wall region of the nest showed significant differences in the properties of the construction elements; the outer nest was composed of significantly thicker, stronger and more rigid elements than the plant materials used in the structural wall or the cup lining (Fig. 2; Table 2). There was no significant effect of nest as a random factor (Table 2). There were also no significant effects of nest region or nest as a random factor for the structural wall $(n=8)$ in the vertical plane on any of the three measured components (Fig. 3; Table 2).

Materials from the top of the nest lining were significantly thinner than in the bottom of the cup (Fig. 4a; Table 2). Materials from the bottom of the cup had significantly higher values for strength and rigidity than materials from the top of the nest cup (Fig. 4b and c; Table 2). Interestingly, for each of the structural elements recovered from the cup lining 'nest' had a significant effect in each model (Table 2).

\section{DISCUSSION}

The materials used in the Common Blackbird nests were similar to those described previously (Bocheński 1968, Simms 1978, Pikula 1983, Mainwaring et al. 2014). Similarly, nest dimensions recorded in this study were comparable to those described in a series of previous 


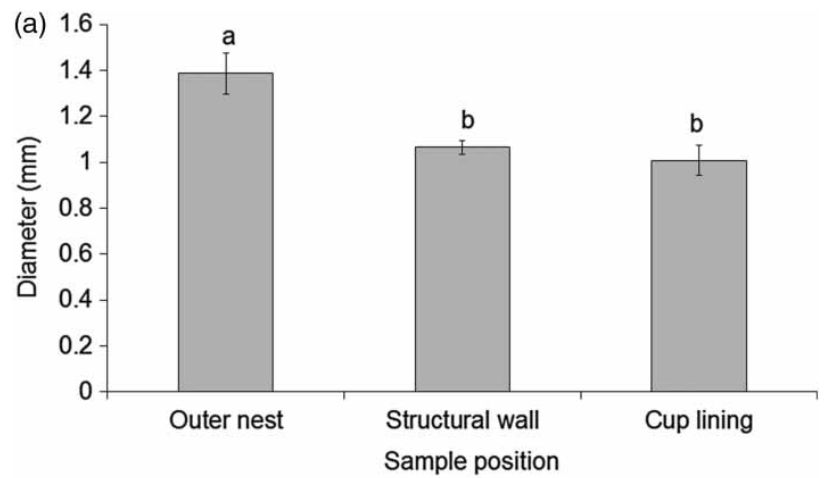

Table 2. Results of general linear modelling to test the effect of location for the three structural measures with nest as a random factor in the model. Values are F-values with the degrees of freedom (df) shown in the row and $P$-values in parentheses.

\begin{tabular}{lcccc} 
& df & Diameter & $\begin{array}{c}\text { Bending } \\
\text { strength }\end{array}$ & Rigidity \\
\hline $\begin{array}{l}\text { Wall region } \\
\text { (Fig. 2) }\end{array}$ & 2,20 & $\begin{array}{c}12.36 \\
(<0.001)\end{array}$ & $\begin{array}{c}12.26 \\
(<0.001)\end{array}$ & $\begin{array}{c}13.13 \\
(<0.001)\end{array}$ \\
$\quad \begin{array}{l}\text { Nest } \\
\text { Structural wall } \\
\text { vertical effect }\end{array}$ & 10,20 & $1.90(0.11)$ & $1.81(0.12)$ & $1.66(0.16)$ \\
$\quad$ Fig. 3) & & $0.76(0.49)$ & $0.66(0.53)$ & $0.79(0.47)$ \\
$\quad$ Nest & 7,14 & $0.50(0.82)$ & $0.85(0.57)$ & $0.81(0.59)$ \\
$\begin{array}{l}\text { Cup lining } \\
\text { vertical (Fig. 4) }\end{array}$ & 1,8 & 34.38 & 19.39 & 18.31 \\
$\quad$ Nest & 8,8 & $(<0.001)$ & $(0.002)$ & $(0.003)$ \\
& & $(0.002)$ & $(0.009)$ & $5.23(0.02)$ \\
\hline
\end{tabular}

known to affect the mass of Great Tit Parus major and Blue Tit nests (Britt \& Deeming 2011, Deeming et al. 2012, Mainwaring et al. 2012).

The volume of the nest cup was estimated as a hemisphere (Møller 1990) and this on average would give a value of $1280 \mathrm{~cm}^{3}$, which was about four-times larger than the actual volumes we measured by filling the space with beads or dental putty. This implies that assuming that the cup is hemispherical is incorrect. Heenan \& Seymour (2011) considered cup shape to be half of a prolate spheroid but whether this represents an accurate estimate of cup shape is not clear. Cup shape in Common Blackbird nests was rather flattened with relatively steep sides and so filling the space with material of known density may be a better way of estimating cup volume than assuming any particular shape.

It was interesting that the use of putty consistently plant material forming the differing nest elements between the regions: (a) diameter $(\mathrm{mm})$; (b) bending strength $\left(\mathrm{N} \mathrm{m} \times 10^{-3}\right)$ and (c) rigidity $\left(\mathrm{N} \mathrm{m}^{2} \times 10^{-6}\right)$. Columns with differing superscripts are significantly different at $P<0.05$.

reports from a variety of European locations (Bocheński 1968, Simms 1978, Pikula 1983, Mainwaring et al. 2012). This tends to imply that nest dimensions, particularly the cup dimensions, are a characteristic of the species concerned (Deeming 2013). The mean mass of the nests in this study was smaller than that reported by Mainwaring et al. (2014) who demonstrated a latitudinal effect on nest mass - nests from Scotland were heaviest with mass decreasing as the nesting location got progressively further south. This difference may reflect differences in location, or perhaps even year of collection. Both factors are produced a bigger volume that the use of acrylic beads. This may be due to differences in methodology but may reflect a compressive effect on the nest structure caused by the heavier putty, which was on average almost two-and-half times heavier than the acrylic beads (567.7 $\mathrm{g}$ versus $208.2 \mathrm{~g}$, respectively). As the putty was being introduced this may have compressed the nest lining materials forcing out air and allowing for more volume of putty. Presumably the magnitude of this effect would differ between nests of different species that utilize differing construction methods and materials, and so is worthy of further study.

It was frustrating that issues regarding mechanical properties of the materials in some of the nests meant that the sample size was reduced from the original 12 samples. It is possible that excluding samples which 

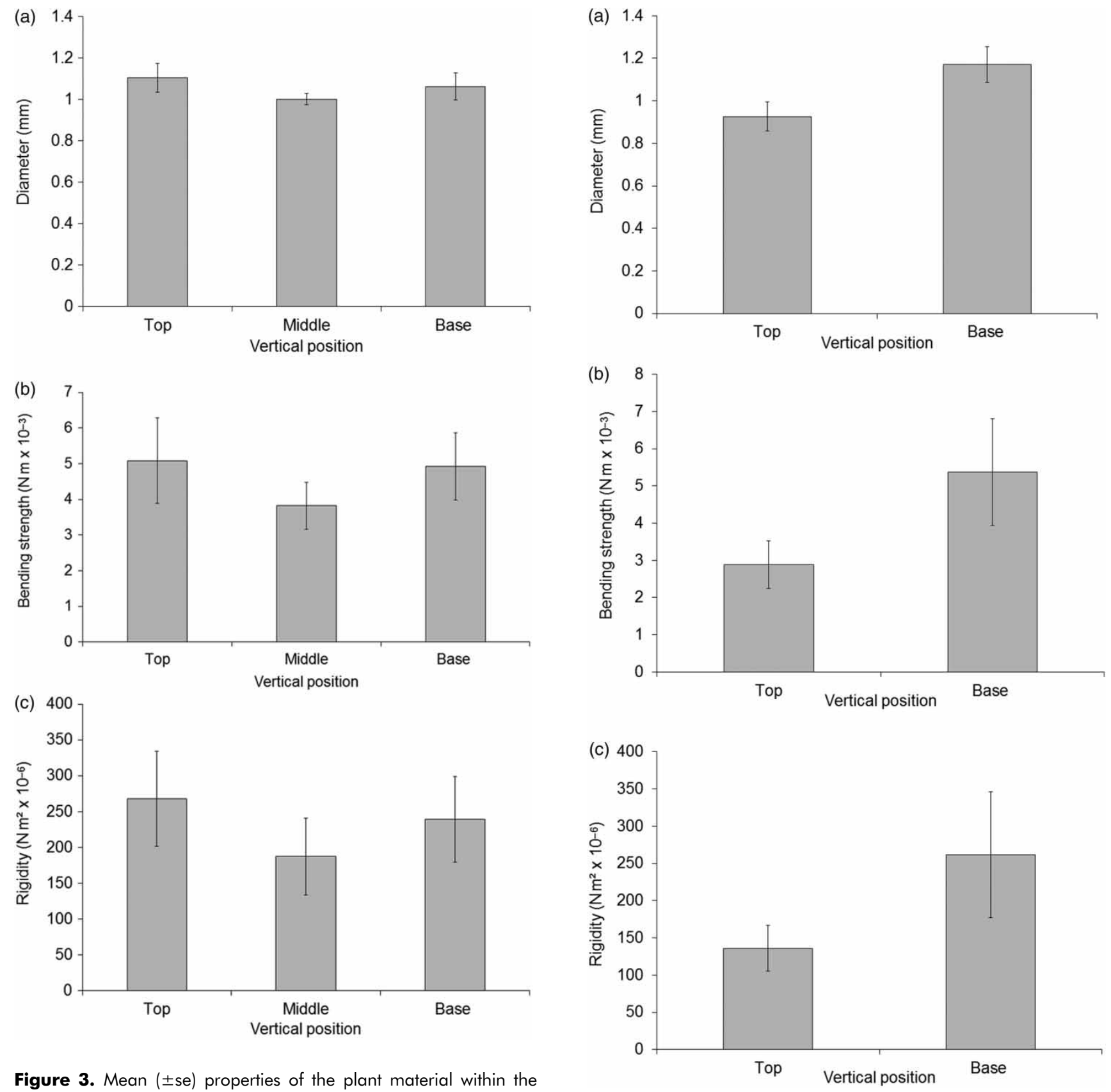

Figure 3. Mean ( $\pm \mathrm{se}$ ) properties of the plant material within the structural wall region in the vertical plane: (a) diameter $(\mathrm{mm})$; (b) bending strength $\left(\mathrm{N} \mathrm{m} \times 10^{-3}\right)$ and $(c)$ rigidity $\left(\mathrm{N} \mathrm{m}^{2} \times 10^{-6}\right)$.

slipped from the supports during mechanical testing led to a potential bias towards nests composed of more uniform construction materials. However, despite small sample sizes, differences that were established were biologically large and statistically significant. Mechanical analysis of the elements within the nest structure suggests that there is limited variation in the properties of the elements within the nest wall. The

Figure 4. Mean ( $\pm \mathrm{se}$ ) properties of the of the plant material forming the lining at the top and base of the cup: (a) diameter (mm); (b) bending strength $\left(\mathrm{N} \mathrm{m} \times 10^{-3}\right)$ and (c) rigidity $\left(\mathrm{N} \mathrm{m}^{2} \times 10^{-6}\right)$. All three comparisons exhibit significant differences at $P<0.01$ (see text for more details).

outer nest clearly was composed of thicker, stronger and more rigid elements which were more loosely arranged. These may have a role in providing a supporting 'foundation' framework for the nest. Elements within the outer nest were around 34\% 
thicker, $152 \%$ stronger and 197\% more rigid than both elements within the structural wall and cup lining regions.

Nests were distinctly elliptical in shape at least in the upper regions yet wall thickness and structural characteristics were the same irrespective of orientation to the long axis of the nest. In other taxa it has been shown that nests are also asymmetrical; in orangutan nests the long axis is orientated pointing towards the tree trunk (van Casteren et al. 2012). The authors suggested that orangutans also select stronger and more rigid branches for the structural parts and weaker more flexible elements for the lining of the nest (van Casteren et al. 2012). It would be interesting to see if blackbirds also position the long axis of their nests in situ relative to the supporting structures in a similar way.

There were, however, significant differences in the properties of elements in the vertical plane of the nest within the cup lining. The elements within the basal region were around $27 \%$ thicker, $87 \%$ stronger and $93 \%$ more rigid than the elements in the top of the nest. The stronger and more rigid elements of the basal area of the cup lining, and the contrasting properties of the elements through the depth of the nest, suggest a mechanical role in better supporting the bird and its eggs from below. This effect also seemed to be specific to individual nests. Mainwaring et al. (2014) showed that the amount of dry grass in the nest cup correlated with the insulatory properties of the nests. It may be possible that different birds select materials of varying properties to line their nests but this requires further investigation.

This study has only considered the properties of the components and not the complexity of the structure itself in which the elements are interwoven and also combined in some cases to form a composite with mud. Our study does however suggest that birds are likely to select nest elements on the basis of diameter, strength and rigidity and that there is an element of positioning within the nest structure in relation to mechanical function; this could have important implications for studies investigating nest building behaviour in birds. Bailey et al. (2014) showed that captive Zebra Finches Taeniopygia guttata preferred string with greater stiffness for their nests, irrespective of their prior experience of the range of string available. Hence, the birds seem to be able to assess the mechanical properties of materials and may choose the most appropriate for their needs.

Mud cups are found within nests of all European species of Turdus (Simms 1978) but we did not measure the mechanical characteristics of the mud-plant composite structure of the inner nest cup because our original intention was to investigate the plant materials, which was incompatible with simultaneously studying the mud cup. We do believe that the mud cup has a key role in maintaining the structural integrity of the nest during incubation and chick rearing. Silva et al. (2010) used finite element analysis on the mud nest of the House Martin Delichon urbicum. They found that its structural properties were complicated by the presence of complex polysaccharides from the bird's saliva and incorporated within the mud wall. It is not known whether saliva is mixed with the mud in blackbird nests, or if it is whether it has a similar role to that seen in House Martins. Further study is required to better characterize this structure.

If the mud cup is a key structural feature that helps maintain integrity of the nest as a whole, why then should the strongest plant materials be found on the outside of the nest? An alternative role for a stronger outer layer of a nest may lie in the construction process. Nest building has not been described in Common Blackbirds but in the closely related Song Thrush Turdus philomelos the earliest stages of construction involves the bird laying out the shape of the nest using substantial plant stems before subsequent material is added to the interior (Goodfellow 2011). Strong, rigid materials around the edge of the nest presumably help support the nest structure until the mud cup is completed, after which the structural properties of the outer layers would be less important. Only careful observation of nest building behaviour in Common Blackbirds will confirm whether this suggestion is applicable to this species' nests. However, it does mean that biomechanical properties of these outer materials, which could possibly be redundant due to structural properties the mud cup, could actually play a crucial role in the construction process rather than maintaining nest integrity after nest completion. Investigation of nests of smaller passerine species that do not incorporate mud may serve useful in developing our understanding of the effect of size on the role of stronger plant elements in the outer layers of a nest.

This study did not consider the thermal properties of the various materials used by the birds, nor how varying the types and amounts of material could impact on the insulatory properties of the nest as a whole. Mainwaring et al. (2014) demonstrated that Common Blackbird nests from different latitudes had varying insulatory properties according to prevailing temperature and that the amount of dried grasses 
correlated with insulatory properties. It is possible that the stiffer, stronger materials used in the bottom of the cup may not only serve to support the eggs and bird but may also trap a greater volume of air, which serves as an insulation layer therefore minimizing heat loss through the bottom of the nest. Stating that nest construction reflects structural roles more than insulation (Heenan \& Seymour 2011) may be oversimplifying the complexity of construction in species that build cup nests. For instance, dry grass was shown by Hilton et al. (2004) to be a relatively poor insulator, compared with feathers or fur. However, animal-derived materials are absent in Common Blackbird nests and dry grass may be relatively important in providing insulation (Mainwaring et al. 2014). Further study is needed to determine how the various nesting materials affect insulation in situ rather than in isolation.

That birds are selecting materials with particular characteristics for specific roles within the structure of their nests is perhaps not surprising. Future work should extend the study to other passerine species of different sizes and that build nests with different materials and in various locations. Moreover, we need to investigate the properties of the nest structure in situ to determine the relative importance of the mechanical behaviour of the overall structure. Future studies could examine the composition and mechanical properties of the mud composite layer. In addition, nest structure and function in closely related species, such as the Song Thrush, or species that build cup nests that are smaller in size, should be investigated from a biomechanical perspective to determine the roles that different materials play during next construction and function.

\section{ACKNOWLEDGEMENTS}

Thanks go to the generous people who collected the nests used in this study, particularly Chris du Feu of the Treswell Wood Ringers Group for his continued support of nest research. Many thanks to Tom Pike, Will Cresswell and an anonymous referee for their constructive comments on a previous version of this manuscript.

\section{REFERENCES}

Álvarez, E., Belda, E.J., Verdejo, J. \& Barba, E. 2013. Variation in Great Tit nest mass and composition and its breeding consequences: a comparative study in four Mediterranean habitats. Avian Biol. Res. 6: 39-46.
Bailey, I.E., Morgan, K.V., Bertin, M., Meddle, S.L. \& Healy, S.D. 2014. Physical cognition: birds learn the structural efficacy of nest material. Proc. R. Soc. B 281: e20133225.

Bocheński, Z. 1968. Nesting of the European members of the genus Turdus Linnaeus 1758 (Aves). Acta Zool. Cracov. 13: 349-439.

Britt, J. \& Deeming, D.C. 2011. First egg date and air temperature affect nest construction in Blue Tits Cyanistes caeruleus but not in Great Tits Parus major. Bird Study 58: 78-89.

van Casteren, A., Sellers, W.I., Thorpe, S.K.S., Coward, S., Crompton, R.H., Myatt, J.P. \& Ennos, A.R. 2012. Nest-building orangutans demonstrate engineering know-how to produce safe, comfortable beds. Proc. Nat. Acad. Sci. 109: 6873-6877.

Collias, N.E. \& Collias, E.C. 1984. Nest Building and Bird Behaviour Princeton University Press, Guildford.

Deeming, D.C. 2013. Effects of female body size and phylogeny on avian nest dimensions. Avian Biol. Res. 6: 1-11.

Deeming, D.C., Mainwaring, M.C., Hartley, I.R. \& Reynolds, S.J. 2012. Local temperature and not latitude determines the design of Blue Tit and Great Tit nests. Avian Biol. Res. 5: 203-208.

Ferguson-Lees, J., Castell, R. \& Leech, D. 2011. A Field Guide to Monitoring Nests. British Trust for Ornithology, Thetford.

Goodfellow, P. 2011. Avian Architecture: How Birds Design, Engineer and Build. Princeton University Press, Princeton, NJ.

Gordon, J.E. 1978. Structures: Or Why Things Don't Fall Down. Penguin Books, London.

Hansell, M.H. 2000. Bird Nests and Construction Behaviour. Cambridge University Press, Cambridge.

Heenan, C.B. \& Seymour, R.S. 2011 . Structural support, not insulation, is the primary driver for avian cup-shaped nest design. Proc. R. Soc. $B$ 278: 2924-2929.

Heenan, C.B. \& Seymour, R.S. 2012. The effect of wind on the rate of heat loss from avian cup-shaped nests. PlosOne 7: e32252.

Hilton, G.M., Hansell, M.H., Ruxton, G.D., Reid, J.M. \& Monaghan, P. 2004. Using artificial nests to test importance of nesting material and nest shelter for incubation energetics. Auk 121: 777-787.

Mainwaring, M.C. \& Hartley, I.R. 2008. Seasonal adjustments in nest cup lining in Blue Tits Cyanistes caeruleus. Ardea 96: 278282.

Mainwaring, M.C., Hartley, I.R., Bearhop, S., Brulez, K., du Feu, C.R., Murphy, G., Plummer, K., Webber, S.L., Reynolds, S.J. \& Deeming, D.C. 2012. Latitudinal variation in blue and great tit nest characteristics indicates environmental adjustment. J. Biogeogr. 39: 1669-1677.

Mainwaring, M.C., Deeming, D.C., Jones, C.I. \& Hartley, I.R. 2014 Adaptive latitudinal variation in Common Blackbird Turdus merula nest characteristics. Ecol. Evol. 4: 841-851.

McGowan, A., Sharp, S.P. \& Hatchwell, B.J. 2004. The structure and function of nests of Long-tailed Tits Aegithalos caudatus. Funct. Ecol. 18: $578-583$.

Moller, A.P. 1990. Nest predation selects for small nest size in the blackbird. Oikos 57: 237-240.

Pikula, J. 1983. Nidobiology of Turdus merula. Acta Sci. Nat. Brno. 17: $1-46$.

Rohwer, V.G. \& Law, J.S.Y. 2010. Geographic variation in nests of Yellow Warblers breeding in Churchill, Manitoba and Elgin, Ontario. Condor 112: 596-604.

Schleicher, B., Hoi, H. \& Valera, F. 1996. Seasonal change in female mate choice criteria in Penduline Tits (Remiz pendulinus). Ardeola 43: 19-29.

Silva, B., Correia, J., Nunes, F., Tavares, P., Varum, H. \& Pinto, J. 2010. Bird nest construction - lessons for building with earth. WSEAS Trans. Environ. Devel. 6: 83-92. 
Simms, E. 1978. The New Naturalist: British Thrushes. William Collins \& Sons, Glasgow.

Skutch, A.F. 1976. Parent Birds and their Young. University of Texas Press, Austin.

Vincent, J.V.F. 1992. Biomechanics-Materials: A Practical Approach. 165-IRL Press at Oxford University Press, Oxford.
Walsh, P.T., Hansell, M., Borello, W.D. \& Healy, S.D. 2010. Repeatability of nest morphology in African weaver birds. Biol. Lett. 6: 149-151.

Walsh, P.T., Hansell, M., Borello, W.D. \& Healy, S.D. 2011 Individuality in nest building: do Southern Masked weaver (Ploceus velatus) males vary in their nest-building behaviour? Behav. Proc. 88: 1-6.

(MS received 12 September 2014; revised MS accepted 4 November 2014) 